\title{
Formación en valores y convivencia escolar mediante la investigación como estrategia pedagógica apoyada en $\mathrm{TIC}^{1}$
}

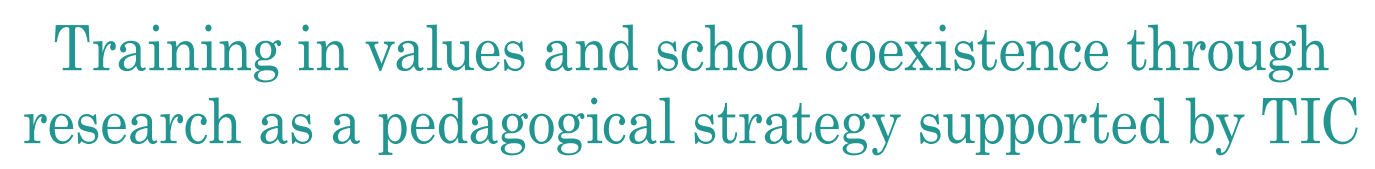

DOI: http://dx.doi.org/10.17981/cultedusoc.9.1.2018.14

Fecha de recepción: 04/05/2018. Fecha de aceptación: 07/08/2018

\author{
Adriana Margarita Mendoza-García ${ }^{2}$ iD \\ Ingrid Esther Blanco-Manjarrez; Damaris Celine Movilla-Villareal ${ }^{3}$
}

Para citar este artículo

Mendoza-García, A., Blanco-Manjarrez, I. y Movilla-Villareal, D. (2018). Formación en valores y convivencia escolar mediante la investigación como estrategia pedagógica apoyada en TIC. Cultura. Educación y Sociedad 9(1), 181-193. DOI: http://dx.doi.org/10.17981/cultedusoc.9.1.2018.14

\section{Resumen}

La formación en valores se ha convertido en una necesidad a nivel educativo, debido a que la escuela además de impartir conocimientos y desarrollar competencias, debe formar en competencias del ser que permitan al individuo tener una adaptación a las normas sociales, ofreciendo de esta manera una educación integral tomando al estudiante como centro del currículo. El estudio tuvo como objetivo fortalecer la formación en valores y convivencia escolar desde la investigación como estrategia pedagógica apoyada en TIC. Se guio bajo el enfoque cualitativo, con un diseño de Investigación Acción Participación (IAP). Donde se utilizaron herramientas como diario de campo y observación participante para la recolección de datos. Se tomó una muestra de cuarenta (40) estudiantes de los grados preescolar a primero de primaria con edades comprendidas entre los cuatro (4) y seis (6) años, del grado preescolar, de la institución educativa Francisco de Paula Santander ubicada en el municipio de Fundación-Magdalena. Las actividades desarrolladas se elaboraron teniendo en cuenta aspectos como la investigación dentro del aula de clases y como proceso de formación, a su vez estas actividades se plantearon teniendo en cuenta las edades de los niños y su contexto.

Palabras clave: formación en valores, convivencia escolar, investigación como estrategia pedagógica, TIC.

\begin{abstract}
Values training has become a necessity at the educational level, because the school, in addition to imparting knowledge and developing competences, must train in competencies of the being that allow the individual to have an adaptation to social norms, thus offering an integral education taking the student as the center of the curriculum. The objective of the study was to strengthen education in values and school coexistence through research as a pedagogical strategy supported by ICT. It was guided under the qualitative approach, with an Investigation Action Participation (IAP) design. Where tools were used as a field diary and participant observation for data collection. A sample of forty (40) students from pre-school to first grade was taken between the ages of four (4) and six (6), of the preschool level of the Francisco de Paula Santander educational institution located in the municipality. of Foundation - Magdalena. The activities developed were made taking into account aspects such as research within the classroom and as a training process, in turn these activities were considered taking into account the ages of children and their context.
\end{abstract}

Keywords: training in values, school life, research as a pedagogical strategy, TIC.

\footnotetext{
${ }^{1}$ Este artículo ha sido derivado del Programa de Fortalecimiento de la Cultura Ciudadana y Democrática CT+I a través de la IEP apoyada en TIC en el Departamento de Magdalena: CICLON

${ }^{2}$ Docente de la IED Francisco de Paula Santander y líder del grupo de investigación Formadoras de valores. Correo de correspondencia: adrianameng@ hotmail.com

${ }^{3}$ Docentes de la institución educativa Francisco de Paula Santander, sede principal, del grupo de investigación Formadoras de Valores.
} 


\section{Introducción}

En efecto, la violencia es un problema que afecta a los hombres y mujeres de modo distinto, transmitida inter-generacionalmente a través de los procesos de socialización, en gran parte responsables de la violencia enfrente a la mujer. La violencia de género es un problema social que tiene serias repercusiones para muchas mujeres, jóvenes y niñas, que incluye ataque físico, social, laboral, psicológica y sexual, entre otras.

Del mismo modo, las investigaciones demuestran que la exposición del hombre a la violencia comienza a una edad temprana en la familia, el sistema educativo y los medios de comunicación, entre otros. En muchos casos, los niños reciben desde muy pequeños el mensaje de que la agresión es un comportamiento socialmente aceptado. Conforme crecen, las dinámicas sociales en torno a la formación de la identidad masculina continúan reforzando la aceptación de una conducta violenta como algo de hombres. Por otro lado, las actitudes socioculturales representan un obstáculo importante para la prevención de la violencia de genero.

Los derechos de la mujer y de la niña tal como establece la Conferencia Mundial de Derechos Humanos (Viena, 1993)- son parte inalienable, integrante e indivisible de los derechos humanos universales. Las violaciones de estos derechos subrayan la estructura de unas relaciones desiguales y muestran la necesidad de comprender que la paz está vinculada a la problemática más amplia de las relaciones no equitativas entre el hombre y la mujer en distintas etapas y ámbitos de su vida personal y social.

La interrelación entre la paz y las relaciones de género es esencial para comprender el alcance de la concepción holística de la paz, que inspira modelos culturales nuevos fundados en la armonía del ser humano consigo mismo (paz interna), con los demás (paz social) y con la naturaleza (paz ecológica). En este sentido, como expone Manchanda (2011), la paz no se concibe como una reinstauración del statu quo, sino que implica la modificación del statu quo de las relaciones de género.

Por ello, la construcción de la cultura de paz a través de la educación, en palabras de Barragán, F.(2012), exige "una revisión no androcéntrica de los mitos que justifican la violencia, así como de las concepciones o creencias que sobre las mujeres tienen los hombres; analizar los materiales curriculares, lo que incluye y lo que excluyen, así como el respeto por la diversidad. La cultura de paz requiere la utilización de estrategias no violentas en las relaciones interpersonales y la revisión crítica del concepto de poder y de los valores. Esto obliga necesariamente a repensar, en términos de mujer y hombre, la idea universal del ser, es decir adquirir conciencia de ser dos y dejar de pensar en lo humano en términos de lo estrictamente masculino.

La escuela, es la institución de socialización segundaria de un individuo, donde se debe realizar una formación integral, que además de las competencias básicas adquiridas en los contenidos curriculares, se fortalezcan las competencias del ser, por lo cual la interiorización de valores se convierte en un aspecto fundamental de la educación, puesto que dicho proceso permitirá la interacción adecuada de estudiantes en cualquier medio que se desenvuelvan, sin embargo, esta es una temática que debe tener un abordaje innovador, puesto que las cátedras tradicionales no presentan un cambio significativo en la población estudiantil, para esto es 
menester utilizar nuevas herramientas, la investigación como estrategia pedagógica es una propuesta constructivista que promueve el aprendizaje en contexto donde el estudiante toma un rol activo en la producción de saber y conocimiento.

La institución educativa Francisco de Paula Santander se encuentra ubicada en el Municipio de Fundación Magdalena, el sector es de estrato socioeconómico bajo y se puede reflejar en la población estudiantil. Los padres de familia se dedican al trabajo informal, una parte numerosa de ellos pertenecen a familias desplazadas por la violencia. En la institución se observan niños y jóvenes con comportamientos agresivos muy marcados y con alta frecuencia vulneran los derechos de sus compañeros, esta situación es evidenciada en los grados escolares iniciales, como preescolar y primero de primaria, los cuales están integrados por niños de cuatro y seis años, en este sentido, la intervención temprana permite un moldeamiento conductual, donde se pueden encontrar resultados positivos a través de la formación en valores, integrando la IEP al currículo.

Ante esta situación, se hace necesario erradicar la violencia en las escuelas, tema que no es fácil, debido a que gran parte de esos problemas están arraizados en las familias, por tal motivo no es tarea fácil, pero esto requiere un compromiso en los docentes, donde deben proponer alternativas de solución que busquen minimizarla, para esto el uso de la investigación es parte importante en el proceso de formación desde los primeros años. Por tal razón, el estudio tuvo como objetivo dirigir una formación en valores desde la investigación como estrategia pedagógica apoyada en TIC. Mediante estas estrategias pedagógicas se busca impactar el currículo impartiendo una formación que permita formar al estudiante de manera integral fortaleciendo la cultura ciudadana en ciencia, tecnología e innovación.

Según Ramírez y Ampudia, (2018). La tecnología, es una herramienta que promueve y aumenta las oportunidades de los países en vías de desarrollo a través del conocimiento. En las instituciones educativas estas han tomado fuerza, puesto que la combinación de información, contexto y experiencia, permiten alcanzar los objetivos de las diferentes áreas de estudio, como medio para obtener y administrar el conocimiento requerido en los estudiantes.

Por lo antes expuesto, es necesario, fortalecer la formación de valores en las familias, en primer lugar, saber escuchar, en comprender racionalmente las razones, ideas y sentimiento de quienes nos hablan, evitando así la intransigencia de las posturas, en segundo lugar, no interrumpir a la otra persona con la que mantenemos un debate o diálogo, para evitar los gritos, la falta de respeto y la tensión y malestar del otro.

Por otro lado,, no atacar las ideas y sentimientos expresados por la otra persona, poniendo en peligro la negociación, asimismo, escuchar de forma activa, realizando preguntas y respuestas constructivas que sirvan para animar al contrario a seguir exponiendo sus ideas y expresando sus sentimientos, para persuadirle de la necesidad de que considere nuestra posición y para hacerle comprender que entendemos lo que nos quiere decir, y por último, utilizar la inducción, de modo que nos anticipemos a la solución que la otra persona nos quiere comunicar, introduciendo las modificaciones necesarias que según nuestro interés posibiliten un acuerdo ventajoso para ambas partes, evitando señales o gestos que impidan 
el acuerdo, favoreciendo aquellos movimientos orientados a indicar a la otra persona que estamos dispuestos a ceder en nuestros intereses, a condición de que ésta ceda también en su postura.

Formación en valores y convivencia escolar: apoyada en la IEP y TIC

La educación en Colombia en los últimos años ha generado transformaciones curriculares, mediante la implementación de diversas estrategias y la capacitación del cuerpo docente de las instituciones estatales, sin embargo, la convivencia escolar se ha convertido en una problemática, por el incremento de agresiones en las instituciones, para lo cual se hace necesario impartir una formación en valores, utilizando la investigación como estrategia pedagógica apoyada en TIC para dinamizar los contenidos programáticos.

La formación en valores implica educar al sujeto para que enfrente las situaciones problemáticas en el mundo social. Cuando los sujetos adoptan una actitud reflexiva frente a los patrones de interpretación cultural que provienen de ese saber de fondo, algún fragmento de la vida se vuelve problemático y se percibe como una situación que demanda acciones e interacciones, las cuales, al ser realizadas, tienen siempre una pretensión de validez y un referente determinado, (Yurén \& De la Cruz, 2016).

Del mismo modo, cuando surge alguna situación en la que algunos valores entran en conflicto, la persona no puede adherirse a un valor, sin más, sino que se ve en la necesidad de reflexionar y determinar cuáles valores acepta como válidos e, idealmente, en virtud de cuáles criterios se da esa aceptación que le permite tomar decisiones. En otras palabras, se pone en cuestión la eticidad prevaleciente y en ese proceso se reflexiona, se pondera, se aplican pautas de valor, y si hay condiciones adecuadas, el sujeto construye principios morales.

Los valores son cualidades peculiares, estos se originan en la relación que se establece, entre el sujeto valorante y el objeto valorado. Sin embargo, es necesario reconocer la existencia de valores con respecto a los actos representativos de la conducta humana, esencialmente de la conducta moral. Todo acto humano implica la necesidad de elegir entre varios actos posibles, pues se prefiere uno a otro acto de acuerdo a lo que se interioriza según cada sujeto como acciones buenas o malas, presentándose como un comportamiento moral, Guevara, B., Zambrano, A., \& Evies, A. (2007).

Por su parte, el termino convivencia hace alusión a la relación que se da entre individuos que conviven o que ocupan un mismo espacio de socialización, este término se utiliza particularmente para hablar del hecho de vivir en armonía, tolerando y respetando las diferencias en un ambiente pacífico, donde se respetan las normas y valores que regulan el trato mutuo, (Pick S, 2002). Sin embargo, la convivencia escolar es un término que ha sido empleado en el ámbito educativo para disminuir los índices de violencia en la escuela, donde se han implementado prácticas autoritarias que reprimen y castigan a los estudiantes, generando violencia estructural, donde el docente o directivos mantienen una relación vertical, adjudicando al estudiante un rol pasivo como en la educación tradicional, Carbajal, P. (2013).

La convivencia en los contextos escolares está precedida por conductas adaptadas y acordes con lo que se espera de los estudiantes escolar y socialmente, hasta otras, que van en dirección opuesta, y que 
terminan en el extremo menos deseable. Dentro de las conductas desaptativas hay que considerar un amplio repertorio conductual con una etiología, sintomatología y pronóstico muy diverso. Todas ellas coinciden en que desestabilizan la convivencia en el aula y en el centro, haciendo más difícil e incluso imposible la consecución de objetivos académicos (De la Fuente; Peralta \& Sánchez, 2006).

Muchas de estas estrategias educativas eficaces tanto en la prevención, como en la intervención, ante el fenómeno del acoso han dado lugar, en España, al establecimiento de programas de educación para la convivencia, que se incluyen en la política educativa de los centros, a veces como líneas transversales de educación en valores, o de forma poco explícita formando parte del llamado curriculum oculto (Boja, 2011). Por otro lado, también desde la literatura científica se han abordado aspectos relativos a la vida social activa en el centro escolar mostrando que tienden a estar relacionados con trabajos sobre políticas globales de intervención (Wong; Cheng; Ngan y Ma, 2010). Esta aproximación holística incluye, al menos, tres elementos; (ver tabla 1).
Para Ramírez, (2018), la gerencia estratégica del talento humano, gestión para la sociedad del conocimiento, y convivencia ante el cambio; está facilitando nuevos estilos administrativos y operativos de labor organizacional para una generación de valor agregado, enfoque sistémico humanizante hacia lo estratégico - dinamizador, inclusión de la ciencia y tecnología, capacidad de internacionalización y asociatividad; convirtiendose en factores claves de competitividad, posibilitando permanencia en el contexto organizacional en un entorno globalizado.

Las estrategias pedagógicas son todas las acciones realizadas por el docente, con el fin de facilitar la formación y el aprendizaje de los estudiantes. Componen los escenarios curriculares de organización de las actividades formativas y de la interacción del proceso enseñanza y aprendizaje donde se logran conocimientos, valores, prácticas, procedimientos y problemas propios del campo de formación.

En la actualidad, existen propuestas metodológicas de enfoques basados en investigación, entendidos como la forma de materializar lo que se hace y como se implementa donde surgen infinidad de

Tabla 1

Aspectos básicos que intervienen en la convivencia escolar: según Ortega, del Rey y Casas, (2013)

\begin{tabular}{cl}
\hline \multicolumn{1}{c}{ Aspecto básico } & \multicolumn{1}{c}{ Definición } \\
\hline Modelos de intervención docente. & $\begin{array}{l}\text { Formas de administrar la instrucción, de establecer normas } \\
\text { y convenciones de relación social, políticas de disciplina o } \\
\text { sanción de las conductas de los escolares, estilos de impartir } \\
\text { enseñanzas, guiar y corregir los aprendizajes de los escolares. }\end{array}$ \\
$\begin{array}{c}\text { Sistema de relaciones } \\
\text { interpersonales de los escolares. }\end{array}$ & $\begin{array}{l}\text { Actitudes de reciprocas entre escolares, agrupamientos } \\
\text { espontáneos, roles y convenciones en el interior de los grupos. }\end{array}$ \\
Elementos perturbadores en el aula. & $\begin{array}{l}\text { Procesos que, si no son atendidos desde la autoridad moral } \\
\text { inseguridad que afecta la convivencia como; fenómenos de } \\
\text { intimidación, abuso, acoso, exclusión social. }\end{array}$ \\
\hline
\end{tabular}

Fuente: elaboración propia, (2018). 
propuestas metodológicas que se basan en un enfoque o toman varios para hacer de ellos una propuesta metodológica; es el caso de las pedagogías basadas o fundadas en investigación, las cuales se desarrollan en diferentes contextos y asumen variados caminos en coherencia con los paradigmas y corrientes en los cuales se inscriben. Dentro de esta teoría basada en investigación se encuentra la Enseñanza por descubrimiento y el Programa Ondas, con su propuesta de la Investigación como Estrategia Pedagógica (IEP), como una perspectiva que se desarrolla en ese enfoque y toma elementos de la pedagogía critico-liberadora y del enfoque socio-cultural, (Mejía, M. \& Manjarrés M, 2010).

De la misma manera, los contenidos del proceso educativo se convierten en problemas, y a partir de preguntas se estructura el currículo, en donde los avances y desarrollos de los estudiantes se determinan por la ampliación de estructura previa que se manifiesta en lenguaje, raciocinios y conocimientos. Ese descubrimiento no es necesariamente autónomo, sino que se hace guiado por el profesor, quien se encarga de planificar los ejercicios y los procedimientos pare el fin buscado, (Pozo, 2006).

El segundo hecho por ser reconocido es el lugar que el trabajo inmaterial (trabajo intelectual) ha tomado en esta sociedad, al convertirse en un generador de valor y, por lo tanto, elemento central para construir el capitalismo de este tiempo, lo cual convierte a actores que trabajan con el conocimiento, la tecnología, la información y la comunicación en sujetos claves de los nuevos procesos de conformación de esta sociedad, alcanzando los educadores de todos los tipos un carácter que organiza y da fundamento a estas modificaciones sociales, (Mejía, 2011).
Es un ejercicio que va a requerir a todo educador, reconocerse y construirse como actor en estas nuevas condiciones, lo cual va a exigir un replanteamiento de las formas anteriores y la consabida modificación de su nueva condición, así como de las transformaciones institucionales en las cuales desarrolla su quehacer. En ese sentido, su subjetividad y los escenarios de su acción se convierten en campos sistematizados y, por lo tanto, en disputa para construir un proyecto de emancipación o de simple modernización al servicio de los grupos dominantes en la sociedad. Es allí donde el educador pone en escena su capacidad humana al servicio de intereses precisos y concretos, (Cetrulo, 2001).

También, en América Latina, así como en grupos subalternos en el mundo del Norte y en diferentes actores críticos de otros continentes, se ha desarrollado, en la perspectiva de los acumulados del paradigma latinoamericano, una concepción de trabajo educativo que se caracteriza por ser una acción política en la esfera de la educación; ella busca transformar las condiciones de control, dominio y formas de sujeción de actores, comunidades e instituciones. Para lograrlo busca generar conciencia crítica y dinámicas sociales que impulsen que los grupos construyan formas de asociación y organización que los convierte en sujetos colectivos, constructores de su historia (Gadotti, 2003).

Allí le da forma a ese otro principio de la educación popular, en la cual la realidad se conoce para transformarla transformándonos a nosotros mismos, y esto desde un horizonte ético que busca develar y enfrentar la segregación, la exclusión y formas de control y dominación que se produce por las múltiples manifestaciones del poder en nuestra sociedad: políticas, económicas, sociales, étnicas, 
en el conocimiento, de subjetividades, de género y en el ejercicio de la práctica educativa y pedagógica; construir un espíritu de emancipación humana, para no desarrollar procesos de poder que controlan ni dominan, ni permitir que otros lo realicen en su propia vida, (Torres, 2007).

En la actualidad el aprendizaje requiere la puesta en acción por parte del estudiante de diferentes competencias que supere la nemotécnica, clave en la escuela de la sociedad postindustrial, y que implica: hacer cosas, pensar sobre conexiones, pensar sobre conceptos, criticar y evaluar, crear conocimiento, y compartir el conocimiento. Ante estas nuevas situaciones creadas por las TIC, las teorías del aprendizaje que tradicionalmente se han movilizado para fundamentar el aprendizaje se presentan como parciales, y se requieren otras nuevas, (Almenara, \& Cejudo, 2015).

Por su parte, la inclusión de las TIC en la educación ha llevado a los docentes a replantearse sus roles, estos influyen directamente en el proceso enseñanzaaprendizaje, y dichos cambios o avances que se han presentado a través del tiempo en el proceso educativo han permitido que se utilicen las herramientas tecnológicas como material de apoyo para el desarrollo de estrategias innovadoras con la finalidad generar motivación en los estudiantes, factor importante y necesario para fomentar un aprendizaje significativo, (Acuña, 2003).

Las TIC son tecnologías para almacenar, recuperar, procesar y comunicar la información. Existe una variedad de recursos electrónicos que se encuadran dentro del concepto de TIC como; televisión, teléfonos, videos, ordenadores, entre otros. Sin embargo, los medios más representativos de la sociedad actual son los ordenadores que nos permiten utili- zar diferentes aplicaciones informáticas: presentaciones, aplicaciones multimedia, programas ofimáticos y redes de comunicación, (Belloch, 2012).

Por otro lado, Avendaño, Cortes \& Guerrero, (2015) expresan que el uso de las tecnologías de la información y la comunicación (TIC) tienen un papel importante en el desarrollo de competencias educativas y sociales, como factores predictores del desempeño académico en estudiantes de básica primaria. Además, las TIC se han convertido en una herramienta innovadora en el contexto educativo generando una relación ineludible que se puede aplicar a cualquier modelo pedagógico a desarrollar y a tener presente de forma trasversal como instrumento pedagógico. (Herrera, 2016).

A lo largo de los planteamientos hechos, se dice, que, la calidad en general del medio socioeducativo es deudora de tres grandes fuentes de influencia: las características del alumnado y de las personas adultas, los aspectos físicos $\mathrm{y}$ organizativos del medio y las condiciones socioeconómicas y sociopolíticas del entorno. Según esto, una institución educativa con una proporción elevada de alumnos "difíciles" y de docentes poco experimentados o desmotivados presentaría un alto riesgo de conflictividad, que afectaría gravemente el clima escolar.

Otros determinantes que aumentarían esta situación serían la ausencia de un medio físico y material adecuado (espacios arquitectónicos inadecuados, déficit de recursos materiales didácticos, y una débil organización (elevada ratio, agrupamientos del alumnado,) y gestión escolar (burocracia, autoritarismo). Como hemos señalado existen relaciones evidentes entre el clima escolar y las prácticas educativas. Según sea la calidad de ambas, los centros estarán más o menos 
Ambiente Socio Cultural.

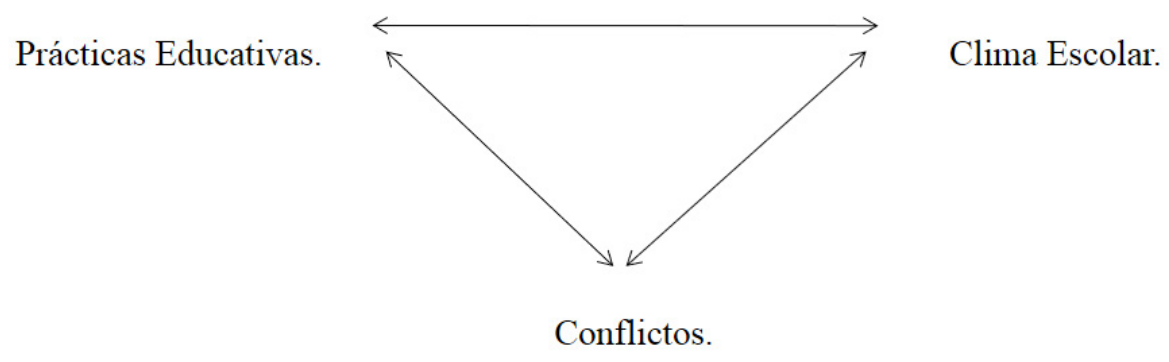

Figura 1. Elementos esenciales para mejorar la convivencia escolar (Tuvilla, 2014). Fuente: elaboración propia, (2018).

propensos a padecer en mayor o menor grado diversos problemas conflictivos. La atmósfera de un centro depende de la naturaleza de las interacciones que se dan en su interior, facilitando o impidiendo la implantación o innovación de ciertas prácticas educativas esenciales para mejorar la convivencia escolar, (ver figura 1).

Pero a su vez la calidad de dichas prácticas afecta también directamente a la calidad del clima escolar. Así, por ejemplo, un centro caracterizado por el uso y abuso de la norma y la sanción para la regulación de la convivencia escolar favorecerá un clima de inseguridad y de injusticia, impidiendo así las prácticas educativas basadas en un aprendizaje cooperativo, debilitando al clima relacional entre el profesorado y el estudiante y deteriorando el clima de pertenencia o adhesión a la institución. De ahí que las relaciones entre el clima escolar y el nivel de conflictividad de un centro se influyan manualmente.

\section{Metodología}

\section{Diseño}

La investigación a realizar en este proyecto es cualitativa, ya que se encarga de comprender la realidad social a través de una observación participante en un contexto. Nuestro contexto el Instituto Francisco de Paula Santander en los grados preescolar. Los estudios cualitativos pueden desarrollar preguntas e hipótesis antes, durante o después de la recolección y el análisis de los datos. Hernández R., Fernández, C. y Baptista, P. (2010). Esto quiere decir, que la investigación cualitativa se encarga de la observación que hacer el investigador, donde recopila toda la información y recoge evidencias, se recogen los datos necesarios y el investigador construye una descripción de lo observado, escribiendo los sucesos del contexto como base para el proyecto de investigación.

En la IAP el conocimiento de la realidad no se descubre ni se posee es el resultado de la trasformación subjetiva y objetiva de el mismo proceso de investigación, en consideración a esta la validez del conocimiento se da por la capacidad que posee para orientar la trasformación de una comunidad o grupo mejorando la calidad de vida de sus integrantes. La IAP es un proceso educativo de autoformación en el cual las personas que pertenecen al grupo sobre quienes esta la realidad estudiada, tienen una participación directa en el proceso de investigación y en la producción de conocimiento de su realidad. 


\section{Participantes}

La unidad de análisis estuvo conformada por cuarenta (40) estudiantes de los grados preescolar a primero de primaria con edades comprendidas entre los cuatro (4) y seis (6) años, del grado preescolar, de la institución educativa Francisco de Paula Santander ubicada en el municipio de Fundación - Magdalena. Cabe resaltar que los participantes diligenciaron adecuadamente el consentimiento informado.

\section{Técnicas e instrumentos}

En cuanto a los instrumentos de recolección se utilizó el diario de campo, este es un instrumento que ayuda al investigador a inmiscuirse de forma total en el ambiente con el cual se va a encontrar, es un proceso de hechos, recolección de datos y análisis. El investigador entiende a los participantes y no se dedica únicamente a registrar hechos. El diario de campo es parte de un registro cronológico que se desarrolla mediante la sesión compartida con los infantes, es donde se describe detalladamente los aspectos positivos como negativos colocando significado a cada uno de ellos como un recurso de mejoras en todas las áreas con el grupo a trabajar considerando los recursos manejados y utilizados. Un cuaderno donde se va a descubrir la manera de expresar y escribir frente a la relación que se tiene con los infantes, es un momento de no cohibir lo vivido y las experiencias obtenidas.

\section{Procedimiento}

La metodología del proyecto de investigación se realizó a partir de los recorridos de las trayectorias de indagación, basa- dos en la integración de la IEP apoyada en TIC al aula, articulado al plan de estudios, desde el área de sociales se estructuraron temáticas relacionadas con la formación en valores y convivencia escolar, la finalidad de este proceso investigativo, fue generar una trasformación social y educativa a través del aprendizaje en contexto, involucrando intereses, motivaciones y realidades de los estudiantes para generar conocimiento científico, donde se establecieron cinco (5) momentos que especifican en la ejecución y las metas trazadas para darle solución a la pregunta problema planteada. A continuación, se presentan los diferentes momentos.

- Momento (1): selección y análisis de la población sujeto de estudio.

- Momento (2): diagnostico poblacional para identificar estilos de aprendizaje y motivación de los estudiantes.

- Momento (3): integración de la temática mediante la IEP al aula de clases, se integraron grupos estudiantiles para seleccionar problemáticas relacionadas con la violencia sexual. Los estudiantes se plantearon preguntas y con el acompañamiento del docente realizaron el proceso de indagación mediante herramientas virtuales y entrevistas a la comunidad.

- Momento (4): socialización de los resultados de las indagaciones de estudiantes en una feria institucional dirigida por los docentes a cargo. y análisis de los resultados de la implementación de la estrategia a través de la observación y diario de campo.

- Momento (5): propagación y divulgación de los resultados. 


\section{Resultados}

A continuación, se presentan las actividades realizadas por los docentes, basadas en juegos y rondas desde la investigación como estrategia pedagógica apoyada en TIC, para fortalecer los valores en los niños y niñas de grado preescolar de la institución educativa departamental Francisco De Paula Santander, ubicada en el municipio de Fundación - Magdalena. (Ver tabla 2)
Teniendo en cuenta el análisis de los instrumentos, como el diario de campo, el diagnóstico inicial mediante la observación de los docentes de preescolar y las actividades ejecutadas, se realiza un análisis crítico en el que se precisa que se debe seguir trabajando en actividades de fortalecimiento de valores en los niños. Las actividades realizadas se llevaron a cabo teniendo en cuenta la edad de los niños, estas actividades respondieron a las necesidades del grupo en la promoción de los valores.

Tabla 2

Actividades pedagógicas para fortalecer los valores escolares

\begin{tabular}{l} 
Actividad \\
\hline Pedro es una Pizza. \\
Se realiza el cuento de Pedro es una \\
pizza. \\
-Realizar inicialmente la masa para \\
que quede como especie de plastilina. \\
-Los niños y niñas escogen a algún \\
compañero para que representen las \\
ilustraciones de Pedro es una pizza. \\
-Cada niño y niña toma una parte de \\
la masa para iniciar la experiencia \\
con la textura y el olor.
\end{tabular}

La Peregrina.

Los niños saltan en orden sobre ella y al llegar al número que cada uno debe llegar, el niño debe expresar un valor.

Proyección de una Película.

$\quad$ Propósito
Motivar a los
niños y las niñas
de preescolar a
tener una relación
adecuada con los
demás niños y
niñas del aula,
fortaleciendo
el respeto, el
compartir y el
trabajo colectivo.

Desarrollar significado de valores a los niños.

$\begin{array}{ll}\text { Objetivo } & \text { Evaluación } \\ \text { Específico } & \end{array}$

Crear

espacios

donde los

niños y

las niñas

fortalezcan el compartir.

Incentivar espacios de aprendizaje, basados en la IEP en donde los niños a través de la investigación descubran el significado de los valores.

Incentivar

Fortalecer el significado de los valores en los niños. espacios de aprendizaje donde los niños identifiquen valores
El proyecto se evaluara a partir de la observación teniendo en cuenta que se logre la motivación como primera medida al ejecutar cada actividad, sus aptitudes y actitudes a la hora de participar en ellas

El proyecto se evaluara a partir de la observación teniendo en cuenta que se logre la motivación como primera medida al ejecutar cada actividad, sus aptitudes y actitudes a la hora de participar en ellas.

El proyecto se evaluará a partir de la observación teniendo en cuenta que se logre la motivación como primera medida al ejecutar cada actividad, sus aptitudes y actitudes a la hora de participar en ellas. 
Juego el Tesoro de los Valores. El tesoro de los valores es escondido por la maestra, se dan pistas para la búsqueda de este tesoro el cual contiene una gran fortuna.

- A cada niño se le brindan pistas, en relación con los tres valores trabajados.

- Al momento que encuentran el tesoro se dicen las palabras mágicas para que se logre abrir.

- Cuando se abre el tesoro de los valores, encuentran demasiadas monedas con los valores trabajados.

Escuchando a los demás.

- Saludo de bienvenida.

Haciendo círculo en el salón se les pregunta a los niños a cerca de cualquier tema en el cual hayan investigado. Se toman 4 o 5 temas en valores para evitar que se repitan las intervenciones de los niños, para ello debe haber silencio y respeto para escuchar a los demás compañeros.

Dramatización con títeres. A través del manejo de títeres se realizara una historia para dar a conocer valores a los niños.

Generar que El proyecto se evaluara a los niños y

Lograr que los niños y las niñas identifiquen el respeto, la generosidad y el compartir como valor. las niñas

reconozcan los valores por medio de monedas de cualquier material. partir de la observación teniendo en cuenta que se logre la motivación como primera medida al ejecutar cada actividad, sus aptitudes y actitudes a la hora de participar en ellas.

El proyecto se evaluara a

Desarrollar significado de

Propiciar espacios de respeto y de escucha. Utilizando de valores a los niños. la IEP en el aula de clases. partir de la observación teniendo en cuenta que se logre la motivación como primera medida al ejecutar cada actividad, sus aptitudes y actitudes a la hora de participar en ellas

El proyecto se evaluará a partir de la observación teniendo en cuenta que

Precisar el significado de valores a los niños.
Ilustrar los valores. se logre la motivación como primera medida al ejecutar cada actividad, sus aptitudes y actitudes a la hora de participar en ellas.

Fuente: elaboración propia, (2018).

\section{Conclusiones}

A partir de este proyecto se trabajó en el aula, impactando el currículo de la institución. La planificación de las actividades se programó a largo plazo para continuar en la construcción de valores; los niños mostraron interés y proactividad en todas las actividades lo cual género en las docentes simpatía para continuar trabajando en las modificaciones que podrían generarse en el currículo a través de proyectos de aula.
Hasta la fecha de estos resultados existen otras actividades que por factores externos no han podido ser realizadas, sin embargo, se estará trabajando en ello. En las actividades se tienen en cuenta elementos investigativos por parte de los alumnos en la construcción de sus conocimientos y poniéndolos en práctica en el aula de clases, esto teniendo en cuenta su edad y contexto. Estos vistos a través de la IEP como estructura principal dentro del aula de clases. 


\section{Referencias bibliográficas}

Acuña, A. (2003). La robótica educativa: un motor para la innovación. Fundación Omar Dengo San José, Costa Rica.

Almenara, J., \& Cejudo, M. (2015). Tecnologías de la Información y la Comunicación (TIC): escenarios formativos y teorías del aprendizaje. Revista Lasallista de Investigación, 12(2), 186-193. Avendaño, I., Cortés, O., \& Guerrero, H. (2015). Competencias sociales y tecnologías de la información y la comunicación como factores asociados al desempeño en estudiantes de básica primaria con experiencia de desplazamiento forzado. Diversitas, 11(1), 13-36.

Barragán, F. (2012). Cultura de paz y violencia de género. En perspectiva, número 5. Sevilla, consejería de Educación y Ciencia

Belloch. (2012). Las Tecnologías de la Información y Comunicación en el aprendizaje. Universidad de Valencia.

BOJA. (2011). Orden de 20 de junio de 2011, promoción de la convivencia en los centros docentes sostenidos con fondos públicos y se regula el derecho de las familias a participar en el proceso educativo de sus hijos e hijas.

Carbajal, P. (2013). Convivencia democrática en las escuelas. Apuntes para una reconceptualizacion. Revista Iberoamericana de Evaluación Educativa, $6(2), 13-35$.

Cetrulo, R. (2001). Alternativas para una acción transformadora: Educación popular, ciencias y política. Montevideo: Ediciones Trilce-Instituto del Hombre.

De la Fuente, J., Peralta, F., \& Sánchez, M. (2006). Valores sociopersonales y problemas de convivencia en la educación secundaria. Electronic Journal of Research in Educational Psychology, 4(9).
Gadotti, M. (2003). Perspectivas actuales de la educación. Buenos Aires: Siglo XXI.

Guevara, B., Zambrano, A., \& Evies, A. (2007). ¿Para qué educar en valores? Revista educación en valores. 1(7).

Hernández, R., Fernández, C. y Baptista, P. (2010). Metodología de la investigación. 5ta Edición. México D.F.: Editorial McGraw Hill.

Herrera-Tapias, B. (2016). Cultura Ciudadana y las Tecnologías de la Información y la Comunicación. Editorial YOYOBIZ. Barranquilla-Colombia.

Machada, R. (2011). Women, War and Peace In South Asia: Beyond victimhood to Agency. New Delhi: Sage Publications.

Mejía, M. \& Manjarrés M. (2010). Las pedagogías fundadas en la investigación. Búsquedas en la reconfiguración de la educación. Revista Internacional Magisterio 42: 16-26.

Mejía, M. (2011). Las escuelas de la globalización. El conflicto por su reconfiguración. Bogotá: Desde Abajo.

Ortega, R., Del Rey, R., \& Casas, J. A. (2013). La Convivencia Escolar: clave en la predicción del Bullying. Revista Iberoamericana de Evaluación Educativa, 6(2).

Pick, S. (2002). Formación cívica y étical Civics and Ethics. Limusa.

Pozo, J. (2006). Aprender y enseñar ciencia. Madrid: Morata.

Ramírez, R. (2018). Tendencias emergentes de la gestión de talento humano en las organizaciones. pp. 101-107. En Quintero Garzón Martha Lucia y Sánchez Fernández María Dolores. (2018) Responsabilidad Corporativa: una mirada integral en América Latina. Primera Edición. Colección Ciencias Sociales. Programa Editorial Universidad del Valle. Cali- Colombia. pp.324. 
Ramírez, R., \& Ampudia, D. (2018). Factores de Competitividad Empresarial en el Sector Comercial. Revista Electrónica de Ciencia y Tecnología del Instituto Universitario de Tecnología de Maracaibo (RECITIUTM). Vol. 4, $N^{\circ}$ 1. Pág.: 16-32.

Torres, A. (2007). La educación popular. Trayectoria y actualidad. Bogotá: El Búho.

Tuvilla, J. (2014). Convivencia escolar y resolución pacifica de conflictos. ISBN: 84-688-5500-6, Deposito legal: SE-1101-04.
Yurén, M. T., \& de la Cruz, M. (2016). La relación familia-escuela: condición de mejora de la eficacia escolar en la formación valoral de niños (as) migrantes. REICE. Revista Iberoamericana sobre Calidad, Eficacia y Cambio en Educación, 7(2).

Wong, D.S.W., Cheng, C.H.K., Ngan, R.M.H. y Ma, S.K. (2010). Program effectiveness of a restorative wholeschool approach for tackling school bullying in Hong Kong. International Journal of Offender Therapy and Comparative Criminology, 55(6), 846-862. 